\title{
Research on Electromagnetic Scattering Properties from Complex Ocean Surface Based on Sea Water Permittivity Model
}

\author{
Liu Wanmeng ${ }^{1}$, Tong Chuangming ${ }^{1}$, Wang Tong ${ }^{1}$ and Liu Zhanqiang ${ }^{1+}$ \\ ${ }^{1}$ Air and Missile Defense College, Air Force Engineering University, Xi'an 710051, China
}

\begin{abstract}
The ocean surface electromagnetic scattering properties have important applications in marine environmental monitoring and detection of target on the sea. The bistatic specular scattering from ocean surface plays a major role in the coupling fields of target and ocean surface. Based on the satellite data of ocean salinity and temperature at the Western Pacific and Southeast coast, the accurate model is built to calculate the permittivity of sea water at different sea areas with foams. The relationships between the foam coverage and air-sea temperature difference and wind speed are analyzed. Based on the modified two-scale model and VRT(vector radiative transfer) theory, the electromagnetic scattering properties from ocean surface with foams at different sea areas are analyzed by using the volume-surface composite model. To make the scattering model conform better to the practice, the cut-off wavenumber at actual wind speed and frequency of incident wave is considered. Then the impacts of foam coverage, wind speed and frequency of incident wave on Brewster effect of ocean surface at different sea areas are further analyzed. The producing mechanism and changing rules of ocean surface Brewster are summarized and analyzed. The research results have important values in military and civil fields.
\end{abstract}

Keywords: ocean surface electromagnetic scattering, specular reflection, Brewster effect, volume-surface composite model

\section{Introduction}

In recent years, the problems of studying the statistical properties of electromagnetic scattering from ocean surface have attached considerable interests in the field of remote sensing and radar surveillance. In the past years, efforts are dedicated to developing more realistic models to describe the complex ocean surface. In [1], a modified two-scale model (MTSM) is presented. The backscattering coefficient of the dynamic ocean surface is calculated by using the modified two-scale model. In [2], the grazing incidence scattering properties from ocean surface with foams at different sea areas are analyzed.

Most of the literatures focus on the analysis of the ocean surface backscattering properties. As for the bistatic specular scattering from ocean surface, there is few of literatures that research on it. However, the specular scattering from ocean surface plays a major role in the mirror interference. In the electromagnetic scattering fields, When the incident wave is vertically polarized wave, the reflection coefficient will attain its minimum at the Brewster angle and the mirror interference will also be the weakest. This phenomenon is called the Brewster effect of rough surface. In [3], the super low level high-parabolic trajectory is designed based on the Brewster angle, and actual flight test proves that it can weaken mirror interference effectively. In [4], it is failed to explain the experiment of Brewster effect by using Small Perturbation Method (SPM) because of the limitation of the method.

In actual situations, part of the ocean surface will be covered with foam when the wind is strong. In view of this, the volume scattering from the foam is incorporated into the modified two-scale model (MTSM), which is called volume-surface composite model. And the cut-off wavenumber at different wind speeds and

\footnotetext{
+ Corresponding author. Liu Wanmeng. Tel.: +18789407671
}

E-mail address:18789407671@163.com. 
frequencies is also considered. The volume scattering from the foam layer is considered by vector radiative transfer (VRT) theory. In this paper, based on the satellite data, the electromagnetic scattering properties and Brewster effect from ocean surface with foams at different sea areas are analyzed by using the volumesurface composite model.

\section{The permittivity model and foam coverage model}

\subsection{The permittivity model of sea water with foams}

The permittivity of sea water has a relationship with the frequency of the incident wave, the salinity and temperature of sea water. The permittivity of sea water can be represented by dual-Debye function as

$$
\begin{aligned}
\varepsilon= & \frac{\varepsilon_{S}(T, S)-\varepsilon_{1}(T, S)}{1+j f / f_{1}(T, S)}+\frac{\varepsilon_{1}(T, S)-\varepsilon_{\infty}}{1+j f / f_{2}(T, S)} \\
& +\varepsilon_{\infty}-j \frac{\sigma(T, S)}{\left(2 \pi \varepsilon_{0}\right) f}
\end{aligned}
$$

Here $T$ and $S$ are temperature and salinity of sea water respectively. In [2], the physical significance of other alphabetic characters are given. In actual situations, part of the ocean surface will be covered with foam when the wind speed is higher. In this paper, the M-G [5] mixed media model is applied to calculating the permittivity of sea with foam.

$$
\varepsilon_{e}=\varepsilon+\frac{3 f_{b}\left(\varepsilon_{p}-\varepsilon\right) \varepsilon /\left(\varepsilon_{p}+2 \varepsilon\right)}{1-f_{b}\left(\varepsilon_{p}-\varepsilon\right) /\left(\varepsilon_{p}+2 \varepsilon\right)}
$$

Here $\varepsilon_{p}$ [6] is the permittivity of foam. $\varepsilon$ is the dual-Debye permittivity of sea water. $f_{b}$ is the empirical formula of foam in sea water.

\subsection{The foam coverage rate}

There are close relationships among the foam coverage rate, wind speed, wind area and the temperature of sea water. In [7], there are several statistic models to calculate the foam coverage rate. When the sea-air temperature difference is moderate, and the atmosphere is stable, the foam coverage rate is presented as

$$
C=2.32 \times 10^{-6} U_{10}^{3.4988}
$$

where $\mathrm{U}_{10}$ is the wind speed in meters per second at an altitude of $10 \mathrm{~m}$ above the sea horizon.

In [8], there is a foam coverage model that includes wind speed and the sea-air temperature difference simultaneously, shown as

$$
C=1.95 \times 10^{-5} U_{10}^{2.55} \exp (0.0861 \Delta T)
$$

where $\Delta T$ is the sea-air temperature difference. The relations among the foam coverage rate, wind speed and the sea-air temperature difference are shown in Fig. 1. We can see from Fig. 1, the foam coverage rate will increase with the increasing of wind speed and the sea-air temperature difference.

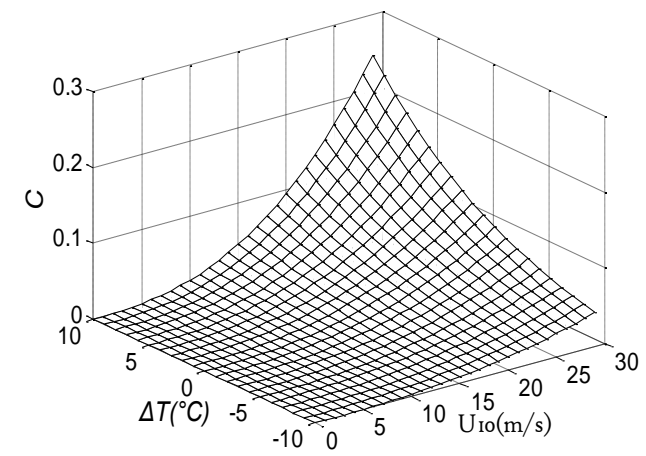

Fig.1: The foam coverage rate

In this paper, we assume the sea-air temperature difference is moderate, and the atmosphere is stable, so the foam coverage rate is calculated by Eq. (3). 


\section{The volume-surface composite model}

\subsection{Modified two-scale theory}

In the modelling of ocean surface scattering, two-scale model (TSM) is widely used. The classical TSM [1] is shown as

$$
\sigma_{p p}=\int_{-\infty}^{\infty} \int_{-\cot \theta_{i}}^{\infty}\left(\hat{p} \cdot \hat{p}^{\prime}\right)^{4} \sigma_{p p}\left(\theta_{s}^{\prime}, \phi_{s}^{\prime} ; \theta_{i}^{\prime}, \phi_{i}^{\prime}\right) \cdot\left(1+z_{x} \tan \theta_{i}\right) P\left(z_{x}, z_{y}\right) d z_{x} d z_{y}
$$

where the subscript $p p$ represents $H H$ or $V V$ polarization. $\sigma_{p p}\left(\theta_{s}^{\prime}, \phi_{s}^{\prime} ; \theta_{i}^{\prime}, \phi_{i}^{\prime}\right)$ is the scattering coefficient derived from the small perturbation method (SPM). $P\left(z_{x}, z_{y}\right)$ is the slope joint probability density function, which can be calculated by Cox-Munk's slick model [9].

When the curvature effect of the surface and shadowing effect are considered, the modified two-scale model (MTSM) [1] can be presented as

$$
\sigma_{p p}=S(v) \cdot \int_{-\infty}^{\infty} \int_{-\cot \theta_{i}}^{\infty}\left(\hat{p} \cdot \hat{p}^{\prime}\right)^{4} c_{p p}\left(R_{x}\right) \cdot\left[\sigma_{p p}\left(\theta_{s}^{\prime}, \phi_{s}^{\prime} ; \theta_{i}^{\prime}, \phi_{i}^{\prime}\right)\right]_{R=\infty} \cdot\left(1+z_{x} \tan \theta_{i}\right) P\left(z_{x}, z_{y}\right) d z_{x} d z_{y}(6)
$$

Here $c_{p p}\left(R_{x}\right)$ is the modified factor of curvature. $S(v)$ is the shadowing function. $P\left(z_{x}, z_{y}\right)$ is the modified slope joint probability density function.

When the wind is strong, part of the ocean surface will be covered with foam which affects the scattering properties and Brewster effect significantly. In order to describe the scattering more accurately, a volumesurface composite model is constructed based on the modified two-scale model (MTSM) and vector radiative transfer (VRT) in this paper. The detailed theory description of the composite model is reported in reference [1]. The brief zero-order and first-order scattering coefficient derived from the zero-order and first-order solution of the VRT equation respectively, shown as

$$
\begin{gathered}
\sigma_{p q}^{0}\left(\theta_{i}\right)=\sigma_{p q 0}\left(\theta_{i}\right) e^{-2 k_{e} d \sec \theta_{i}} \\
\sigma_{h h}^{1}\left(\theta_{i}\right)=\frac{3}{4} \cos \theta_{i} \frac{k_{s}}{k_{e}}\left(1-e^{-2 k_{e} d \sec \theta_{i}}\right)+\frac{3}{4} \cos \theta_{i} \frac{k_{s}}{k_{e}}\left|R_{h h}\right|^{4} \times e^{-2 k_{e} d \sec \theta_{i}}\left(1-e^{-2 k_{e} d \sec \theta_{i}}\right) \\
\sigma_{v v}^{1}\left(\theta_{i}\right)=\frac{3}{4} \cos \theta_{i} \frac{k_{s}}{k_{e}}\left(1-e^{-2 k_{e} d \sec \theta_{i}}\right)+\frac{3}{4} \cos \theta_{i} \frac{k_{s}}{k_{e}}\left|R_{v v}\right|^{2} \times e^{-2 k_{e} d \sec \theta_{i}}\left(1-e^{-2 k_{e} d \sec \theta_{i}}\right)
\end{gathered}
$$

Here $k_{s}$ and $k_{e}$ are the scattering and extinction coefficients of the foam particles. $\sigma_{p q 0}\left(\theta_{i}\right)$ is the scattering coefficient of the rough foam-free ocean surface. $e^{-2 k_{e} d \sec \theta_{i}}$ is the attenuation factor when the EM wave propagates in the layer of foam particles with thickness $d . R_{h h}$ and $R_{v v}$ are the Fresnel reflection coefficients of flat surface for horizontal and vertical polarization, respectively.

The sea surface is covered with foam partly under strong wind, so the total scattering coefficient is contributed by the sea surface with and without foam, shown as

$$
\sigma_{p q}=(1-C) \sigma_{p q 0}\left(\theta_{i}\right)+C \times\left(\sigma_{p q}^{0}\left(\theta_{i}\right)+\sigma_{p q}^{1}\left(\theta_{i}\right)\right)
$$

\subsection{The selection of cut-off wavenumber}

In the two-scale model, the long gravity type sea waves and short capillary type sea waves are connected by the parameter cut-off wave number $k_{c}$. In the past research, the cut-off wavenumber will be given based on the electromagnetic wave number, and it is definitive in a range of sea conditions. However, the cut-off wave number should change when the wind speed or the frequency of incident wave changes. Hence, to get more accurate numerical results, the cut-off wavenumber at different wind speeds and frequencies is considered based on the established conditions of large-scale and small-scale sea waves.

The established condition of the large-scale sea waves component is shown as

$$
2 \pi \sigma_{c} / k_{i} \ll 1
$$

where $k_{i}$ is the incidence electromagnetic wave number. $\sigma_{c}$ is the standard deviation of ocean surface mean curvature, calculated as

$$
\sigma_{c}^{2}=\int_{0}^{k_{c}} k^{4} W(k) d k
$$


where $W(k)$ represents the sea spectrum.

The established condition of the small-scale sea waves component is shown as

$$
k_{i} \delta_{s} \cos \left(\theta_{i}\right) \ll 1
$$

where $\theta_{i}$ is the incidence angle. $\delta_{s}$ is root-mean-square height of small-scale sea surface, calculated as

$$
\delta_{s}^{2}=\int_{k_{c}}^{\infty} W(k) d k
$$

Based on the established conditions of large-scale and small-scale sea waves, the cut-off wavenumber at different wind speeds and frequencies is presented as

$$
\frac{4 \pi^{2}}{k_{i}^{2}} \int_{0}^{k_{c}} k^{4} W(k) d k=k_{i}^{2} \cos ^{2}\left(\theta_{i}\right) \int_{k_{c}}^{\infty} W(k) d k
$$

In Fig. 2, curves of relationship between parameter $k_{c} / k_{i}$ and wind speed at five different wavebands are shown. Here the incidence angle $\theta_{i}=10^{\circ}$. We can see from Fig. 2, the specific value of the cut-off wave number and the incidence electromagnetic wave number will decrease first and then increase with the increase of wind speed. In the high frequency ( $\mathrm{X}$ band and $\mathrm{K}_{\mathrm{u}}$ band), the specific value will only decrease slightly at low wind speed.

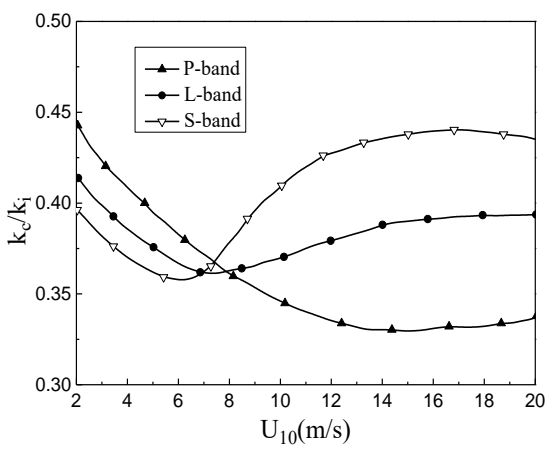

(a) P band, $\mathrm{L}$ band and $\mathrm{S}$ band

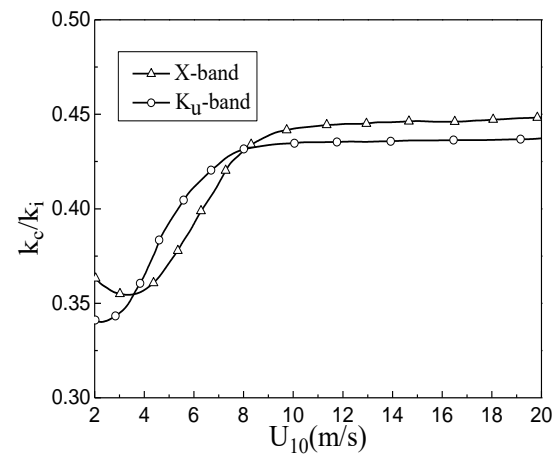

(b) $\mathrm{X}$ band and $\mathrm{K}_{\mathrm{u}}$ band

Fig.2: The relations between the cut-off wavenumber and wind speed and frequency

\section{Numerical results and discussion}

In this paper, the ocean surface geometric model is built based on the Elfouhaily sea spectrum. And the area of the geometric model is $128 \mathrm{~m} \times 128 \mathrm{~m}$. The comparisons of the backscattering coefficient predicted by the volume-surface composite model and MTSM with the experimental data are shown in Fig. 3 and Fig. 4. The experimental data is from SASS-II pentrough [10]. The temperature of the sea water is the average value $17.4^{\circ} \mathrm{C}$. The salinity of the sea water is the global average salinity value $32.54 \%$. In Fig. 3, the foam coverage rate is $0.732 \%$. We can see from Fig. 3, at large incidence angles, the volume-surface composite model has a better agreement with the experimental data than the MTSM. The simulation results demonstrate the validity of the composite model. We can see from Fig. 4, the volume scattering of the foam becomes conspicuous with the increase of the incidence angle and the wind speed, which results in the heightening of the level of the composite model curve.

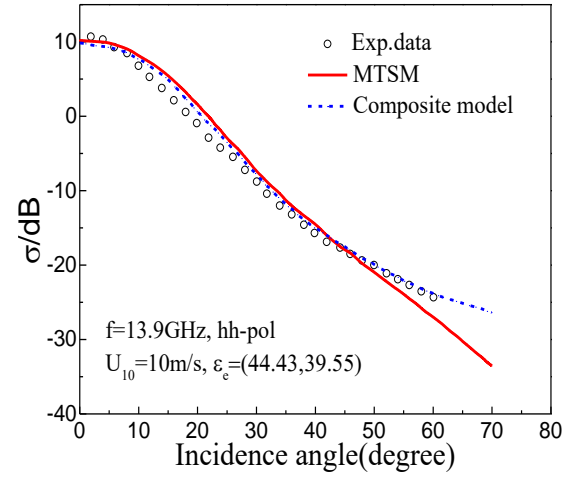

Fig.3: Demonstration of the volume-surface composite model

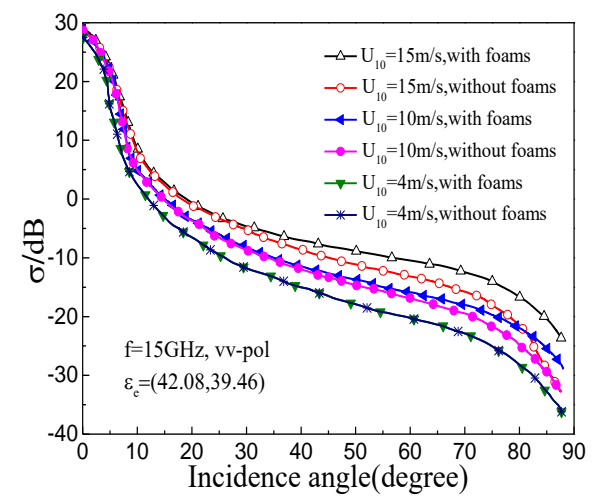

Fig.4: Comparisons of the two models 
When the $V V$ polarization electromagnetic wave projects from air to the smooth earth surface or ocean surface, the amplitude ratio between the reflected wave and incident wave is called reflection coefficient, which can be calculated by Fresnel formula.

In actual situations, the ocean surface is rough, so the reflection coefficient can't be calculated by Fresnel formula. So we use the volume-surface composite model to research the impacts of foam coverage, wind speed and frequency on the Brewster effect at different sea areas, shown in Fig. 5, the ordinate is the absolute value of reflection coefficient. We can see from Figs. 5(a), (b) and (c), the Brewster effect will weaken with the increase of wind speed and the frequency of incident wave. The reason is that the ocean surface will be rougher with the increase of wind speed and the frequency, and it strengthens the incoherent scattering and weakens the specular scattering. With the increase of wind speed, the foam coverage becomes larger, and it results in the discrepancy between with foams and without foams becomes conspicuous. The Brewster effect of ocean surface will weaken because of the presence of foams. Observing the curve of Brewster effect carefully, we can find that the Brewster angle will shift with the changing of wind speed.

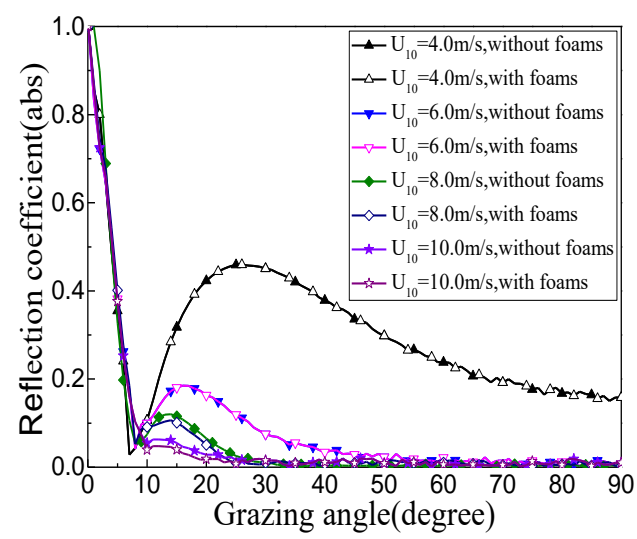

(a) The Brewster effect in $\mathrm{P}$ band

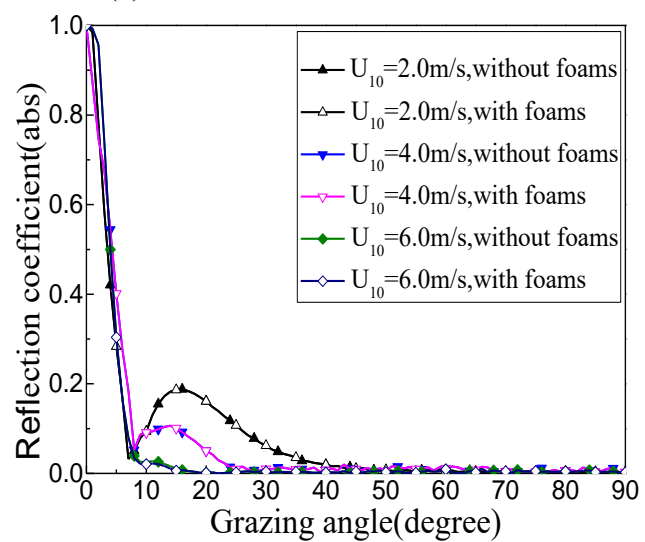

(c) The Brewster effect in X band

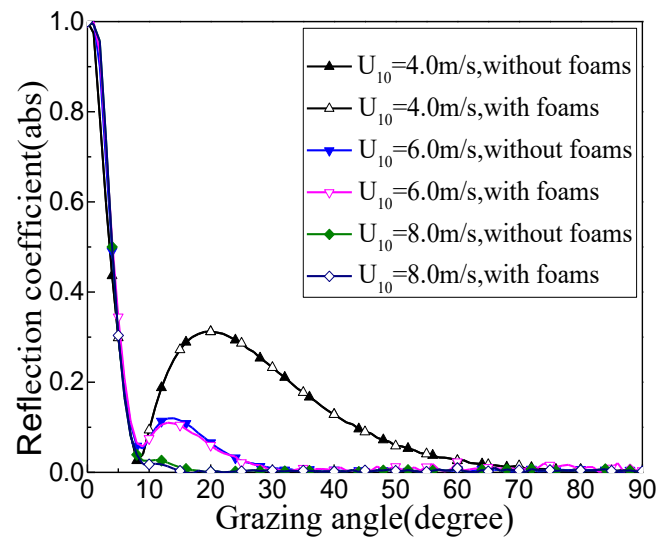

(b) The Brewster effect in L band

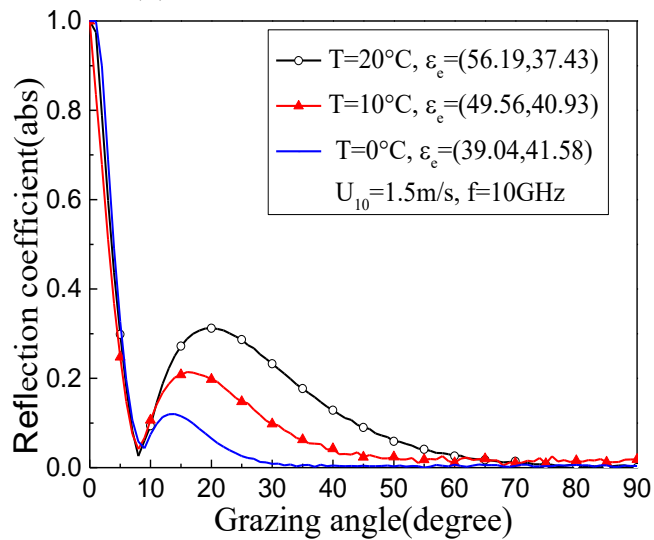

(d) The Brewster effect at different temperatures

Fig.5: Analysis of the Brewster effect of ocean surface

Based on the satellite data from Ocean University of China, the average temperature and salinity at different sea areas in August, 2013 is shown in table 1.

Table 1 Average temperature and salinity at different sea areas in August, 2013

\begin{tabular}{lcccc}
\hline Waters features & Bohai Sea & Yellow Sea & East China Sea & South China Sea \\
\hline Temperature $/{ }^{\circ} \mathrm{C}$ & 25.0 & 22.6 & 27.7 & 29.1 \\
Salinity/ \%0 & 33.5 & 31.1 & 33.6 & 33.5 \\
\hline
\end{tabular}

We can see from table 1, the change of ocean salinity in different sea areas is very small. So we use the change in temperature to represent the different sea areas, shown in Figs. 5(d). It is obvious that the real part of the permittivity will decrease with the fall of temperature, and it results in the weakening of the specular scattering. Hence, from Figs. 5(d) we can see that the Brewster effect of ocean surface will weaken with the decreasing of the temperature. 


\section{Conclusions}

The accurate permittivity model of sea water with foams and the foam coverage model are constructed. Based on the satellite data of ocean salinity and temperature at the Western Pacific and Southeast coast, the electromagnetic scattering properties and Brewster effect from complex ocean surface are investigated by the volume-surface composite model. And the cut-off wavenumber at different wind speeds and frequencies of incident wave is considered. We can arrive at some conclusions as the following:

1) The wind speed, frequency of incident wave and the temperature of sea water will all influence the electromagnetic scattering properties and Brewster effect from ocean surface.

2) At high wind speed, the volume scattering of the foam becomes conspicuous, and it will influence the electromagnetic scattering properties and Brewster effect obviously.

3) The Brewster angle will shift with the changing of wind speed and frequency of incident wave. However, the changing rules of ocean surface Brewster angle are not discussed in this paper, and it can be researched in the future.

\section{References}

[1] Z. S. Wu, J. P. Zhang, and L. X. Guo. An improved two-scale model with volume scattering for the dynamic ocean surface. Progress in Electromagnetics Research. 2009, 89:39-56.

[2] T. $\mathrm{Wu}, \mathrm{L} . \mathrm{X}$. Linghu, etal. Elements affecting low-grazing-angle electromagnetic scattering properties from ocean surface at multi-band in different sea area. Chinese Journal of Radio Science. 2015, 30(6):1078-1085.

[3] X. C. Yang, L. Sun, etal. Study of super low level high parabolic trajectory design andsimulation of ground-to-air missile weapon system. Journal of Projectiles, Rockets, Missiles and Guidance. 2010, 30(3):47-52.

[4] J.-J.Greffet. Theoretical model of the shift of the Brewster angle on a rough surface. Optics Letters. 1992, 17(4):238-240.

[5] A. H. Sihvola, J. A. Kong. Effective permittivity of dielectric mixtures. IEEE Transactions on Geoscience and Remote Sensing. 1988, 26(4): 420-429.

[6] K. Kuang, M. Chang. The model and application of sea electromagnetic scattering. Acta Oceanologica Sinica. 1992, 14(5): 34-44.

[7] M. H. Smith, P. M. Park, I. E. Consterding. Marine aerosol concentration and estimated fluxes over the sea. Q. J. R. Meteorol. Soc. 1993, 119 (512): 809-824.

[8] E. C. Monahan, I. C. O'Muircheartaigh. Whitecaps and the passive remote sensing of the ocean surface. Int. J. Remote sensing. 1986, 7 (5): 627-642.

[9] S. T. McDaniel, Microwave backscatter from non-Gaussianseas. IEEE Trans. Geosci. Remote Sens. 2003, 41(1):52 - 58.

[10] A. G. Voronovich, V. U. Zavorotni. Theoretical model for scattering of radar signals in Ku- and C- bands from a rough see surface with breaking waves. Waves Random Media. 2001, 11(3): 247-269. 